\title{
A rare case of gastric injury during routine laparoscopic surgery
}

\author{
Ziena Abdullah • Akobundu Nnochiri
}

Received: 30 April 2011 / Accepted: 31 May 2011 / Published online: 29 June 2011

(C) Springer-Verlag 2011

Keywords Laparoscopy · Gastric injury · Laryngeal mask airway $\cdot$ Gastric distension

\section{Introduction}

Over the last two decades, laparoscopic surgery has indeed become the primary method of choice for the diagnosis and management of most gynaecological conditions. This is rightly so as randomised controlled trials have shown that the risk of minor complications after gynaecological surgery is $40 \%$ lower with laparoscopy, compared to laparotomy [1]. However, worldwide studies have revealed that the rate of major laparoscopic complications may be increasing, as more complicated and difficult procedures are being performed. These complications maybe in the form of bowel and vascular injuries particularly during the creation of a pneumoperitoneum, which is rarely seen in open surgery. Here, we report a rare case of gastric perforation during laparoscopic sterilisation.

\section{Case presentation}

A 38-year-old multiparous woman with three spontaneous vaginal deliveries and one caesarean section for

Z. Abdullah $(\bowtie)$

Queens Hospital, Rom Valley Way, Romford,

Essex RM7 0AG, UK

e-mail: z_abdullah83@yahoo.co.uk

A. Nnochiri

Rom Valley Way, Romford,

Essex RM7 0AG, UK twins was admitted for laparoscopic sterilisation. She had a history of depression and had attempted suicide in 2002. She smoked 40 cigarettes per day and occasionally consumed large amounts of alcohol. Her last menstrual period was 3 years ago possibly due to her low BMI of 17 (weight $41 \mathrm{~kg}$ ).

Following detailed preoperative counselling and confirmation of the consent, general anaesthesia was induced and a laryngeal mask was used to maintain the airway. She was then placed in the modified Lloyd Davies position with the use of the stirrups. She was cleaned and draped and the bladder was emptied. A uterine manipulator was inserted vaginally as required. A 5-mm intra-umbilical incision was made and a Veress needle was inserted without any difficulty. Palmer's test was satisfactory. At a pressure of $20 \mathrm{mmHg}$, a 5 -mm umbilical trocar was inserted using the standard technique. After insertion of the trocar, it was noticed that the initial intraperitoneal pressures were between 12 and $15 \mathrm{mmHg}$. The trocar was removed, and a 5-mm visiport was used to gain entry. The camera was introduced, and it was immediately recognised that a lumen had been entered. The gas was turned off and the camera was withdrawn but the trocar was left in place, and the general surgeons were asked to attend. The anaesthetist, at this point, replaced the laryngeal mask with an endotracheal tube and aspirated some fluid from the stomach using a nasogastric tube.

With the general surgeon present, the camera was reinserted into the peritoneal cavity and a thorough inspection of the abdominal contents revealed two perforations on the anterior wall of the stomach each measuring approximately $0.5 \mathrm{~cm}$ in length and dense omental adhesions to the anterior abdominal wall were present. The laparoscopic sterilisation was then performed with one filshie clip to each fallopian tube. Gastroscopy was also 
performed, which ruled out concomitant injury to the posterior wall of the stomach. The lacerations were repaired by intracorporeal suturing. Omental adhesions were separated, and the abdominopelvic cavity was irrigated using warm saline. The remainder of the procedure was completed without any further complications. Postoperatively, the patient was placed on antibiotics and was discharged 2 days later. She had an unremarkable postoperative period and was seen in the outpatient clinic after 3 weeks.

\section{Discussion}

Laparoscopy is a relatively safe procedure, however, complications can occur, and are related predominantly to trocar or Veress needle insertion. The incidence ranges from 0.84 per 1,000 for minor laparoscopic surgery, 4.30 per 1,000 for major surgery and 17.45 per 1,000 for advanced laparoscopic surgery [2]. A thorough review of the literature found that the commonest sites of injury are urological, vascular and gastrointestinal, which has an incidence of 0.62 and 1.60 per thousand laparoscopies [2] and a complication rate of $20-46 \%[2,3]$. However, an injury to the stomach is rare. Chapron et al. [4] found only one gastric injury in a retrospective study of 56 patients of whom 62 gastrointestinal injuries were identified during gynaecological laparoscopy. Here, we present a rare case of gastric injury sustained during abdominal entry using the closed technique routinely used by gynaecologists.

In the few reported cases of gastric injury, various mechanisms of damage have been described. Mateus et al. [5] postulated that their case was likely due to gastric dilatation caused by their patient's peristaltic dysfunction of the colon, while Milliken and Milliken believed the cause for their case was due to the insertion of the Veress needle $2 \mathrm{~cm}$ above the umbilicus, patient anxiety and prolonged manual oxygenation by the anaesthetist [6]. Whilst in our case, the injury may have been due to the choice of anaesthetic airway management.

The choice of anaesthetic technique for laparoscopic surgery is predominantly general anaesthesia. However, penetration of the stomach may occur when the stomach is distended during the induction of anaesthesia. Some reports have implicated the use of a laryngeal mask as a cause for gastric distension, particularly if it is unknowingly malpositioned, and therefore an independent risk factor for gastric injury [7]. A laryngeal mask was used in our patient, and we believe that this may have been responsible for the hyperinflation of the stomach causing it to descend to a level below the umbilicus and in the path of the Veress needle and trocar. Care needs to be taken to avoid overinflating the stomach during ventilation. Where this is suspected to have occurred, due to upper abdominal distension or increased tympanism, a nasogastric tube should be inserted to evacuate the stomach prior to the creation of pneumoperitoneum in order to prevent injuries to the anterior surface of the stomach [4]. Should a complication occur, early recognition is essential.

Most complications are missed during the surgical procedure. In a study by Chapron et al. [2], almost a third of complications $(28.6 \%)$ went unnoticed intraoperatively and were only diagnosed subsequently. Those overlooked were $23.7 \%$ for urological complications, $20 \%$ for vascular complications and $41.8 \%$ for bowel injuries. Early recognition of injury is essential for a more favourable outcome. Once recognised, intestinal or stomach injuries should be treated immediately, as a delay could lead to significant mortality and morbidity. In our case, the injury was recognised intraoperatively and repaired by laparoscopy. If bowel injury is suspected, it is essential to leave the Verress or trocar in situ to aid in the identification of the site of injury. Alternative visual access can be created by the insertion of a 5-mm suprapubic trocar, if a gastric injury is suspected, or in the left hypochondrium (Palmer's point [8]) if an intestinal injury is suspected. This should be followed by a systematic inspection of the entire abdominopelvic cavity. When identified, the injury should be repaired without any tension preferably by operative laparoscopy, but only if the surgeon is adequately skilled in laparoscopic suturing and possesses suitable equipment to carry out the procedure. It would be prudent to seek the advise of a laparoscopic gastrointestinal surgeon in such cases. However, if the injury is large, with significant intraabdominal spillage of bowel contents, conversion to open laparotomy should be performed.

\section{Conclusion}

In conclusion, our case is a unique example of a rare laparoscopic complication, which all surgeons should be aware of and have the duty to warn their patients of. Most importantly, as in our case, the injury was recognised early and treated swiftly at the time of the procedure. Such cases may be prevented by having a high index of suspicion for the possibility of gastric distension. If suspected, insert a nasogastric tube to decompress the stomach prior to creating the pneumoperitoneum and use the correct surgical techniques to avoid the stomach.

Conflicts of interest The authors report no conflicts of interest. The authors alone are responsible for the content and writing of the paper. 


\section{References}

1. Chapron C, Fauconnier A, Goffinet F, Breart G, Dubuisson JB (2002) Laparoscopic surgery is not inherently dangerous for patients presenting with benign gynecologic pathology: results of a meta-analysis. Hum Reprod 17:1334-1342

2. Chapron C, Querleu D, Bruhat MA et al (1998) Surgical complications of diagnostic and operative gynecologic laparoscopy: a series of 29 966 cases. Hum Reprod 13:867-872

3. Peterson HB, Hulka JF, Phillips JM (1990) American Association of Gynecologic Laparoscopists' 1988 membership survey on operative laparoscopy. J Reprod Med 35:587-589
4. Chapron C et al (1999) Gastrointestinal injuries during gynaecological laparoscopy. Hum Reprod 14(2):333-337

5. Mateus J, Pezzi C, Stephen G (2006) Recognition and prevention of gastric injury during gynecologic laparoscopy. Obstet Gynecol 108:804-806

6. Milliken RA, Milliken GM (1975) Gastric perforation: rare complication of laparoscopy. N Y State J Med 75:77-79

7. Roger Maltby J, Beriault MT, Watson NC, Fick GH (2000) Gastric distension and ventilation during laparoscopic cholecystectomy: LMA-classic vs. tracheal intubation. Can J Anesth 47 (7):622-626

8. Palmer R (1974) Safety in laparoscopy. J Reprod Med 13:1-5 